\title{
A Hybrid Approach for Fast Computation of Multiple Incident Angles Electromagnetic Scattering Problems with Compressive Sensing and Adaptive Cross Approximation
}

\author{
Guo-hua Wang and Yu-fa Sun \\ Key Laboratory of Intelligent Computing \& Signal Processing, Ministry of Education, Anhui University, Hefei 230039, China \\ Correspondence should be addressed to Yu-fa Sun; yfsun_ahu@sina.com
}

Received 30 October 2015; Revised 15 February 2016; Accepted 9 March 2016

Academic Editor: Renato Cicchetti

Copyright (C) 2016 G.-h. Wang and Y.-f. Sun. This is an open access article distributed under the Creative Commons Attribution License, which permits unrestricted use, distribution, and reproduction in any medium, provided the original work is properly cited.

\begin{abstract}
A hybrid compressive approach for fast computation of the electromagnetic scattering problems with multiple incident angles is proposed. The compressive sensing (CS) technique is firstly introduced to the method of moment (MoM) to reduce the number of the right-hand sides (RHS), but since the resulting excitation matrix contains linear dependency and has low-rank characteristics, the adaptive cross approximation (ACA) algorithm is used to recompress such excitation matrix, keeping only the necessary physical information. The hybrid compressive approach can reduce the number of the RHS to lower level. In fact, the ratio of the number of the RHS between the compressed excitation matrix and the original excitation matrix in the conventional MoM is close to one to fifteen. Numerical results are presented to validate the efficiency and accuracy of this method, which turns out to be highly efficient and accurate for the solution to multiple incident angles electromagnetic scattering problems.
\end{abstract}

\section{Introduction}

Accurate and efficient analysis of the electromagnetic scattering with multiple incident angles has attracted increasing attention from academia and industry. The method of moment (MoM) $[1,2]$ together with iterative methods is often used for the accurate analysis of electromagnetic scattering problems, and some fast algorithms like the multilevel fast multipole algorithm (MLFMA) [3], the adaptive integral method (AIM) [4], and the adaptive cross approximation (ACA) $[5,6]$ are generally applied to reduce both the computation time and the memory requirements for electrically large radiation and scattering problems. But, for the problems with multiple incident angles, the above methods become time-consuming due to the presence of multiple right-hand side (RHS) problems.

An efficient method to accelerate the solution to multiple RHS problems is to remove redundancies in the initial excitation and reduce the number of RHS. In $[7,8]$, the compressive sensing (CS) theory $[9,10]$ was introduced into the traditional MoM to solve wide-angle monostatic scattering problem of 2D targets. In [11], the CS technique was used to analyze the multistatic scattering problems. In this paper, a new excitation matrix is firstly constructed with smaller RHS number than traditional MoM, and the measurement values of the induced current coefficients can be calculated by direct method or iterative method. Finally, true current coefficients can be reconstructed by a recovery method such as the orthogonal matching pursuit (OMP) [12] algorithm. However, the number of the measurement values and sparse transformation base in the CS are difficult to determine according to different scattering targets. In [7], to balance the computational complexity and the accuracy, the number of measurements was set to 24 . To reduce the number of measurements to a lower level than the CS technique, a hybrid compressive method named CS-ACA is advised. Such method utilizes the low-rank characteristics of the new excitation matrix generated by the CS. The CS technique is firstly used with a reduced number of RHS; then the ACA algorithm is introduced into CS to recompress the new excitation matrix so as to reduce the number of RHS further, so as to realize the efficient calculation of the multiple excitation $3 \mathrm{D}$ electromagnetic problems. 


\section{A Hybrid Compressive Technique of the Excitation Matrix}

2.1. Compressive Sensing Theory. The CS theory exploits the inherent scarcity or compressibility features of the signal in some transform basis and directly samples the most important information of the signal at the rate far below Nyquist rate [13]. The original signal can be reconstructed accurately by solving an optimization problem.

Let $\mathbf{x} \in \mathbf{R}^{R}$ represent a discrete signal of length $R$, which can be expanded as a linear combination of a set of standard orthogonal bases $\Psi=\left[\psi_{1}, \psi_{2}, \ldots, \psi_{R}\right]$ :

$$
\mathbf{x}=\boldsymbol{\Psi} \cdot \boldsymbol{\alpha}=\sum_{i=1}^{R} \psi_{i} \alpha_{i}
$$

where $\psi_{i}$ is a set of basis functions in orthogonal basis $\Psi$ and $\boldsymbol{\alpha}=\left[\alpha_{1}, \alpha_{2}, \ldots, \alpha_{R}\right]^{T}$ is the expanded complex coefficients vector of signal. $T$ represents the transpose operation. If $\mathbf{x}$ can be represented by combination of only $K$ nonzero entries and $K \ll R$, it can be named $K$-sparse [14] in $\Psi$ field. Assume that $\mathbf{x}$ is one-dimensional signal of length $R$ and $M$ is the number of measurements. By constructing $M \times R(M \approx$ $K \ln (R / K) \ll R)$-dimensional measurement matrix $\Phi$ and computing $M$ inner products operation, we can obtain the observation signal $\mathbf{y}$, which is the linear measurement of the original signal $\mathbf{x}$. $\mathbf{y}$ can also be seen as the projection of a sparse signal in a low dimensional space:

$$
\mathbf{y}=\boldsymbol{\Phi} \cdot \mathbf{x}=\boldsymbol{\Phi} \cdot \Psi \cdot \boldsymbol{\alpha}=\mathrm{A}^{\mathrm{CS}} \cdot \boldsymbol{\alpha},
$$

where the CS matrix is defined as $M \times R$-dimensional matrix $\mathbf{A}^{\mathrm{CS}}$. Once the measurement $\mathbf{y}$ has been obtained, a reconstruction algorithm is required to reconstruct the sparse signal $\boldsymbol{\alpha}$ from the measured vector $\mathbf{y}$ of length $M$. As the number of measurements is much smaller than the dimension of the original signal, the reconstruction process becomes an issue of solving the underdetermined equations, which generally has no determined solution. However, if $\mathrm{A}^{\mathrm{CS}}$ satisfies strict restricted isometry property (RIP) [15] condition, this issue will have a determined solution, and the signal recovery issue can be converted to convex optimization algorithm; then the approximation of $\boldsymbol{\alpha}$ can be obtained by solving the $l_{1}$-norm minimization problem:

$$
\begin{aligned}
\widehat{\boldsymbol{\alpha}}=\min & \|\boldsymbol{\alpha}\|_{1} \\
\text { s.t. } & \mathbf{y}=\mathbf{A}^{\mathrm{CS}} \cdot \boldsymbol{\alpha} .
\end{aligned}
$$

Finally, the original signal can be reconstructed as

$$
\widehat{\mathbf{x}}=\Psi \cdot \widehat{\boldsymbol{\alpha}} .
$$

2.2. Application of CS to Multiple Excitation Problems. In this paper, the MoM in the frequency domain is used to solve $3 \mathrm{D}$ electromagnetic scattering problems by solving the related electric field integral equations (EFIEs). By using Rao-Wilton-Glisson (RWG) vector basis functions [16] and employing Galerkin's method of moments, the related EFIEs can be transformed into a dense, complex system of linear equations:

$$
\mathbf{Z} \cdot \mathbf{J}=\mathbf{V}
$$

where $\mathbf{Z} \in \mathbf{C}^{N \times N}$ is the impedance matrix and $N$ is the number of unknowns. $\mathbf{V} \in \mathbf{C}^{N \times R}$ is the excitation matrix with $R$ plane waves and $\mathbf{J} \in \mathbf{C}^{N \times R}$ is the induced current density matrix. For various angles of incidence, computing responses became time-consuming due to the presence of a multiple RHS problem. In order to reduce the number of RHS and accelerate the solution process, according to CS theory [7-11], new excitation is constructed as follows:

$$
\mathbf{V}_{m}^{\mathrm{CS}}=\sum_{r=1}^{R} \phi_{m r} \mathbf{V}\left(\theta_{r}\right), \quad m=1,2 \cdots M
$$

in which the new source is formed by random superposition of the incident at different incident angles. Substituting $\mathbf{V}^{\mathrm{CS}}$ into (5),

$$
\mathbf{Z} \cdot \mathbf{J}_{m}^{\mathrm{CS}}=\mathbf{V}_{m}^{\mathrm{CS}}, \quad m=1,2 \cdots M
$$

in which $\mathbf{V}_{m}^{\mathrm{CS}}$ is the column vector of new excitation matrix $\mathbf{V}^{\mathrm{CS}}$. As the impedance matrix $\mathbf{Z}$ is not changed with incident angle, each column vector of $\mathbf{J}_{m}^{\mathrm{CS}}$ can be described as

$$
\mathbf{J}_{m}^{\mathrm{CS}}=\sum_{r=1}^{R} \phi_{m r} \mathbf{J}\left(\theta_{r}\right), \quad m=1,2 \cdots M .
$$

Rewriting $\mathbf{J}_{m}^{\mathrm{CS}}$ and $\mathbf{J}\left(\theta_{r}\right)$ as $M$-dimensional row vector, (8) can be expressed as a matrix form

$$
\begin{aligned}
\mathbf{J}^{\mathrm{CS}} & =\left(\begin{array}{c}
\mathbf{J}_{1}^{\mathrm{CS}} \\
\mathbf{J}_{2}^{\mathrm{CS}} \\
\vdots \\
\mathbf{J}_{M}^{\mathrm{CS}}
\end{array}\right) \\
& =\left(\begin{array}{cccc}
\phi_{11} & \phi_{12} & \cdots & \phi_{1 R} \\
\phi_{21} & \phi_{22} & \cdots & \phi_{2 R} \\
\vdots & \vdots & \ddots & \vdots \\
\phi_{M 1} & \phi_{M 2} & \cdots & \phi_{M R}
\end{array}\right)\left(\begin{array}{c}
\mathbf{J}\left(\theta_{1}\right) \\
\mathbf{J}\left(\theta_{2}\right) \\
\vdots \\
\mathbf{J}\left(\theta_{R}\right)
\end{array}\right)=\mathbf{\Phi} \cdot \mathbf{J}
\end{aligned}
$$

in which $\Phi$ is $M \times R$-dimensional Gaussian random number matrix. Actually, $\mathbf{J}^{\mathrm{CS}}$ can just be seen as the $M$-dimensional measurements of true induced current coefficients J. On the other hand, according to CS theory [7-11], $\mathbf{J}^{\mathrm{CS}}$ can also be written as

$$
\mathbf{J}^{\mathrm{CS}}=\boldsymbol{\Phi} \cdot \boldsymbol{\Psi} \cdot \boldsymbol{\alpha}=\mathbf{A}^{\mathrm{CS}} \cdot \boldsymbol{\alpha}
$$

in which $\mathbf{A}^{\mathrm{CS}}$ is defined as CS matrix. Once the expanded complex coefficients matrix $\boldsymbol{\alpha}$ has been calculated by OMP, J can be reconstructed accurately. As the induced current is $K$ sparse in orthogonal basis $\Psi$ and the number of measurement values $M \ll R$, the number of RHS in linear system equations is reduced. 
2.3. Recompression of the RHS Excitation Matrix. As stated in previous works [7,8], the new excitation matrix $\mathbf{V}^{\mathrm{CS}}$ is formed by random superposition of various plane waves with different directions of incidence, which is of low rank and can be recompressed by a low-rank representation [17] algorithm to reduce the RHS to lower level than that by using CS method.

An efficient technique to achieve a matrix low-rank approximation is ACA algorithm, which has been introduced by Bebendorf in [5]. In 2005, the algorithm has been successfully applied to moderate electrical size scattering and radiation computations [6]. The ACA algorithm is purely algebraic in nature, kernel independent, and relatively easy to implement. Also, the algorithm does not require a priori knowledge of each impedance submatrix.

The new excitation matrix $\mathbf{V}^{\mathrm{CS}}$ can be well approximated by the product of two full-rank matrixes using ACA method:

$$
\mathbf{V}^{\mathrm{CS}} \approx \mathrm{V}_{U}^{\mathrm{CS}} \cdot \mathbf{V}_{V}^{\mathrm{CS}}
$$

where $\mathbf{V}_{U}^{\mathrm{CS}} \in \mathbf{C}^{N \times \mathrm{Q}}$ and $\mathbf{V}_{V}^{\mathrm{CS}} \in \mathbf{C}^{\mathrm{Q} \times R}$ and $Q$ is the effective rank of new excitation matrix which is much smaller than the number of RHS. Induced current density matrix $\mathbf{J}^{\mathrm{CS}}$ in (9) can be calculated approximately by exploiting the following expression:

$$
\mathbf{J}^{\mathrm{CS}} \approx\left(\mathbf{Z}^{-1} \cdot \mathbf{V}_{U}^{\mathrm{CS}}\right) \cdot \mathbf{V}_{V}^{\mathrm{CS}} .
$$

This expression reduces the computational effort massively, because the number of RHS is equal to the rank $Q$, which is much smaller than the initial number of RHS in (5).

\section{Numerical Results}

To test the performance of the new method, the scattering characteristics of differently shaped objects are presented and compared with those obtained by using the traditional MoM solution. The measurement matrix $\boldsymbol{\Phi}$ in the CS is set to Gaussian random matrix, orthogonal basis $\Psi$ is set to discrete cosine transform matrix, the OMP is used as the recovery algorithm, and the ACA iteration error threshold is set to $1 e-4$. Both examples are calculated on a PC with $3 \mathrm{GHz}$ Intel Core 2 Duo CPU and 8 GB RAM (only one core is used). To provide a comparison between the original and the recovered current coefficients, the following relative root mean square error (RRMSE) is introduced:

$$
\text { RRMSE }=\sqrt{\frac{1}{N} \sum_{i=1}^{N} \frac{\left|I_{i}^{\text {Recovered }}-I_{i}^{\text {original }}\right|^{2}}{\left|I_{i}^{\text {original }}\right|^{2}} .}
$$

3.1. NASA Almond Model. The scattering problem of a 252.3744 mm perfect electric conductor (PEC) Almond [18] at the frequency of $7 \mathrm{GHz}$ is considered first. The surface of the Almond has been discretized by RWG basis functions with average edge length of $\lambda / 10$, which requires 12621 unknowns. The model is illuminated by a set of incident plane waves with incident direction of $\varphi \in\left[0^{\circ}, 180^{\circ}\right]$ and $\theta=90^{\circ}$, where the angle interval for $\varphi$ is $1^{\circ}$. The monostatic

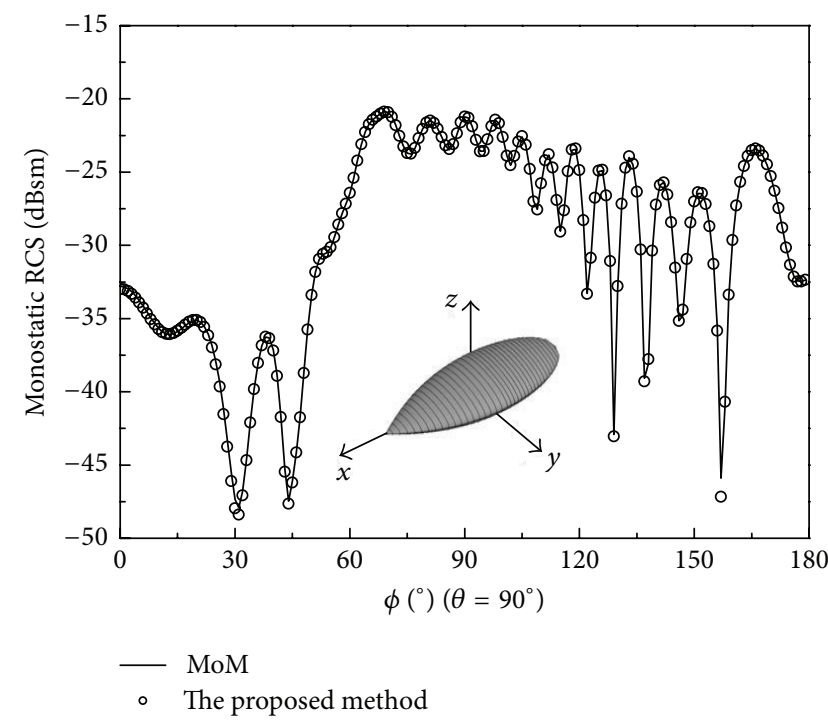

FIgURE 1: Monostatic RCS of PEC Almond model.

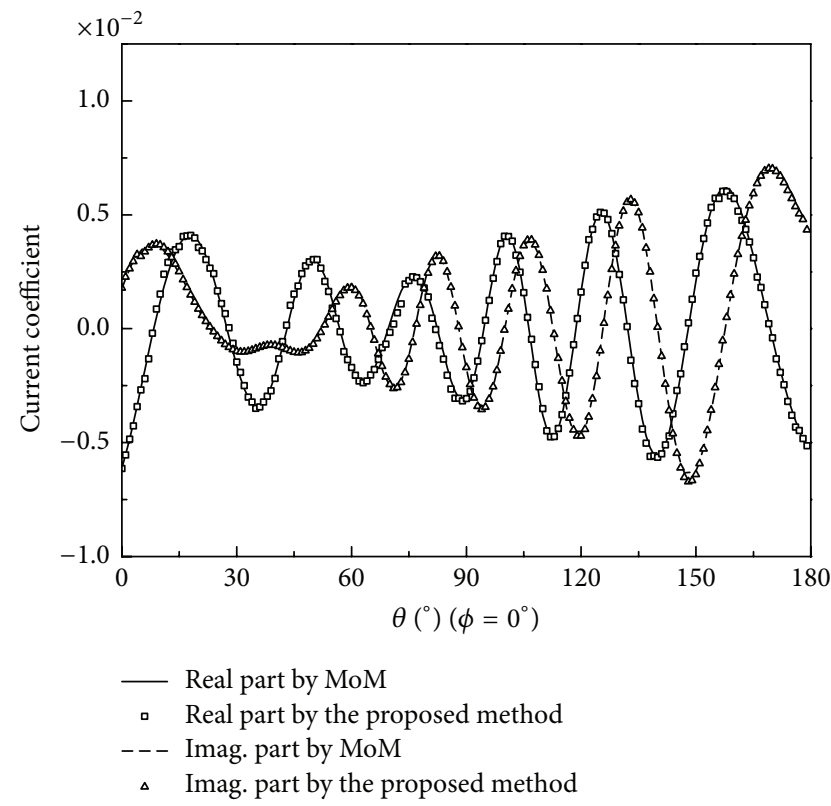

FIGURE 2: True and recovery current coefficients for Almond model.

radar cross section (RCS) is computed by using the CS-ACA method proposed in this paper. The CS technique is firstly applied with the measurement number $M=40$, and then the new excitation matrix $\mathbf{V}^{\mathrm{CS}}$ is recompressed by using the ACA, which can further reduce the number of RHS from $M=40$ to $\operatorname{rank} Q=12$. The backscattering monostatic RCS in horizontally transmitted and horizontal received $(\mathrm{HH})$ field polarization calculated by the CS-ACA method and the traditional MoM are shown in Figure 1.

The recovered current coefficients and the true current coefficients (computed by MoM) obtained using a RWG basis with multiple incident angles are shown in Figure 2.

As the effective rank of $\mathbf{V}^{\mathrm{CS}}$ takes different values, the RRMSE varies with it. The relationship between the global 


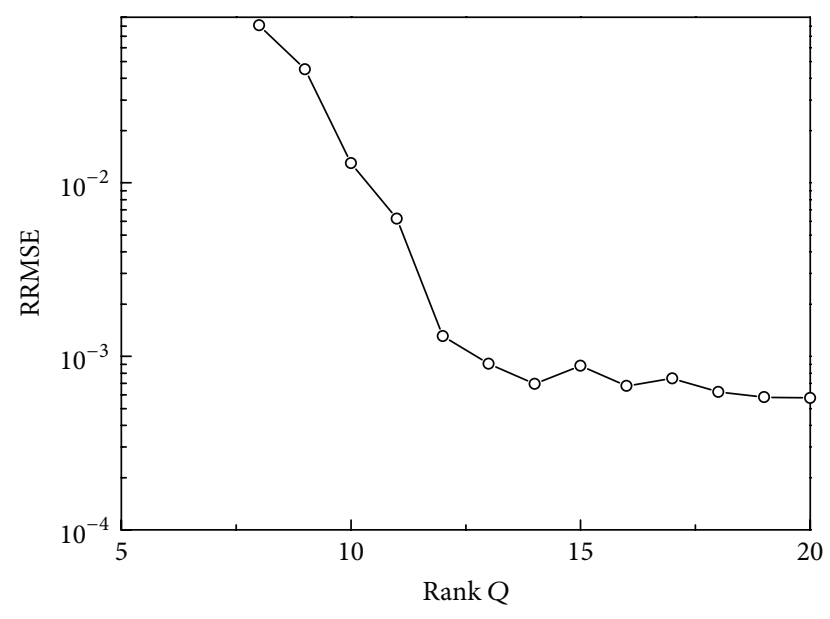

$\multimap-$ RRMSE of CS-ACA

FIGURE 3: RRMSE of CS-ACA method for Almond.

RRMSE and the effective rank is presented in Figure 3, from which we can find that the CS-ACA method can reduce the number of RHS effectively with very low RRMSE.

High numerical accuracy of the current distributions makes it possible to obtain a reliable value of the near-field distribution, as shown in [19], where the field generated by the surface currents lying on a Cartesian quadrant has been assessed in closed analytical form to evaluate the exact field distribution excited by current pulse basis functions. The spatial distribution of the electric field component at a distance $\lambda_{0} / 1000$ above the Almond, computed using the proposed hybrid approach, is shown in Figure 4.

3.2. PEC Missile Model. The second example is a PEC missile model shown in Figure 5, which has a length of $9.91 \mathrm{~m}$, a wingspan of $6.28 \mathrm{~m}$, and a height of $2.19 \mathrm{~m}$.

The HH polarization backscattering monostatic RCS is computed at the frequency of $300 \mathrm{MHz}$, which requires 25263 unknowns. The missile model is illuminated by a set of incident plane waves with the incident direction of $\theta \in$ $\left[0^{\circ}, 180^{\circ}\right]$ and $\varphi=0^{\circ}$, where the angle interval for $\theta$ is $1^{\circ}$. Firstly, the number of measurements in the CS is set to $M=40$, and then the new excitation matrix generated by the CS is recompressed by the ACA, which can further reduce the RHS number to $Q=14$. The $H H$ polarization backscattering monostatic RCS in $x$ - $z$-plane calculated by the CS-ACA method in this paper and the MLFMA are shown in Figure 4. Recovered and true current coefficients obtained through using a RWG basis are shown in Figure 6, while the relationship between global RRMSE with rank $Q$ of $\mathbf{V}^{\mathrm{CS}}$ is shown in Figure 7. From this figure, it appears that the RRMSE is still very low when rank $Q$ is greater than 14 . From the above examples, we can conclude that the results obtained by the CS-ACA method are very accurate.

Table 1 lists the CPU time requirements of the proposed examples. It can be seen that the CS-ACA method has compressed much more CPU time demand than the conventional MoM implementation.

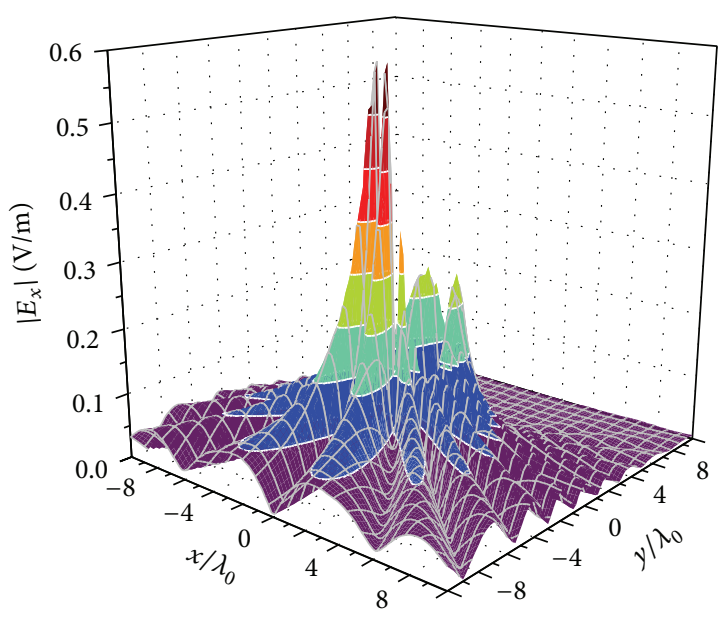

(a)

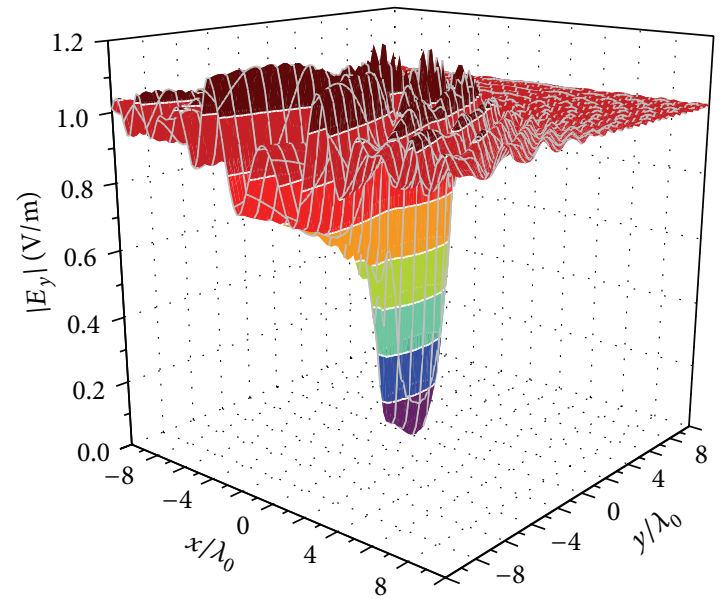

(b)

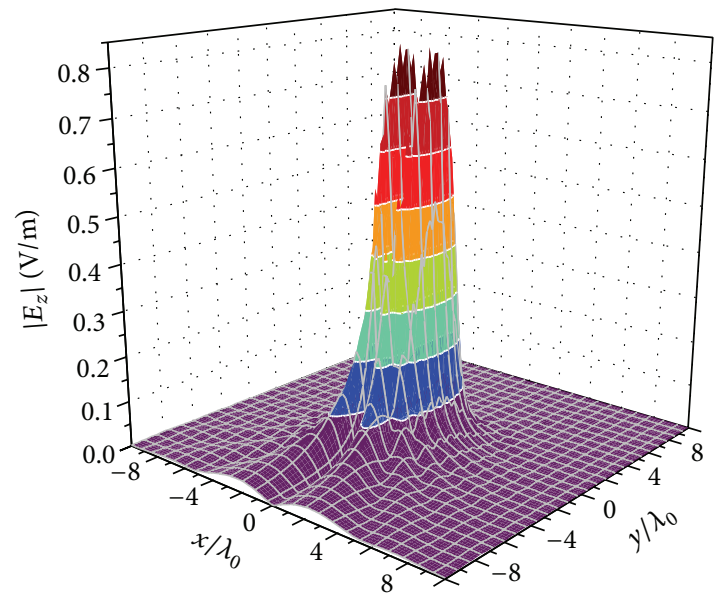

(c)

Figure 4: Magnitude of the total electric field $\lambda_{0} / 1000$ above the Almond: (a) $x^{-}$-, (b) $y$-, and (c) $z$-component of the electric field. Characteristics of the incident field: $E_{0}=1 \mathrm{~V} / \mathrm{m}, \theta_{i}=90^{\circ}, \varphi_{i}=0^{\circ}$, and polarization angle $\alpha=90^{\circ}$.

\section{Conclusions}

An efficient method to analyze the electromagnetic scattering problems with multiple incident angles based on the 
TABLE 1: CPU time requirements.

\begin{tabular}{lccc}
\hline Target & Method & $\begin{array}{c}\text { RHS } \\
\text { number }\end{array}$ & Time/s \\
\hline PEC NASA Almond & MoM & 180 & 1564 \\
& CS-ACA & 12 & 257 \\
\hline \multirow{2}{*}{ PEC missile model } & MLFMA & 180 & 765 \\
& CS-ACA & 14 & 436 \\
\hline
\end{tabular}

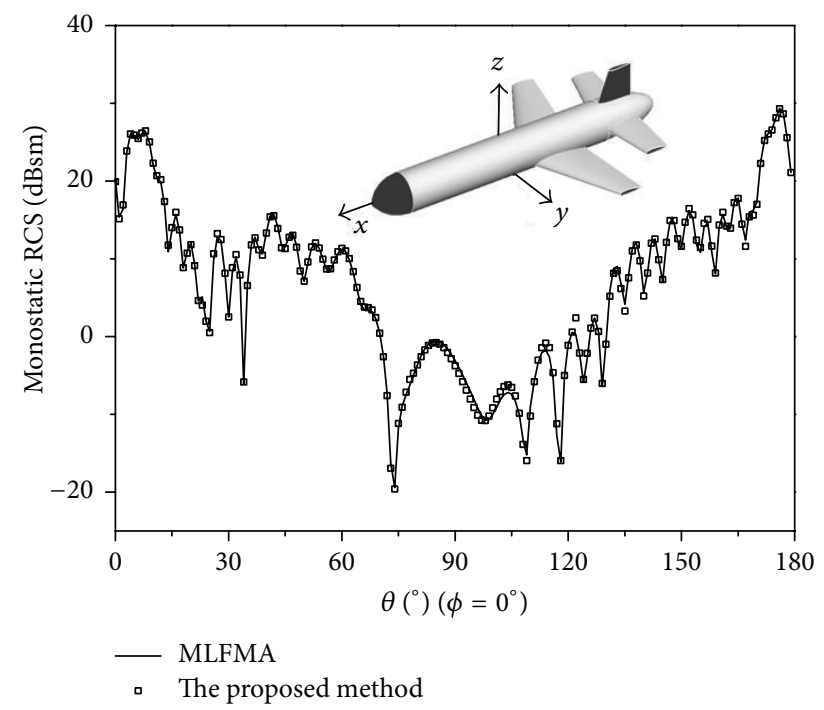

FIgURe 5: Monostatic RCS of PEC missile model.

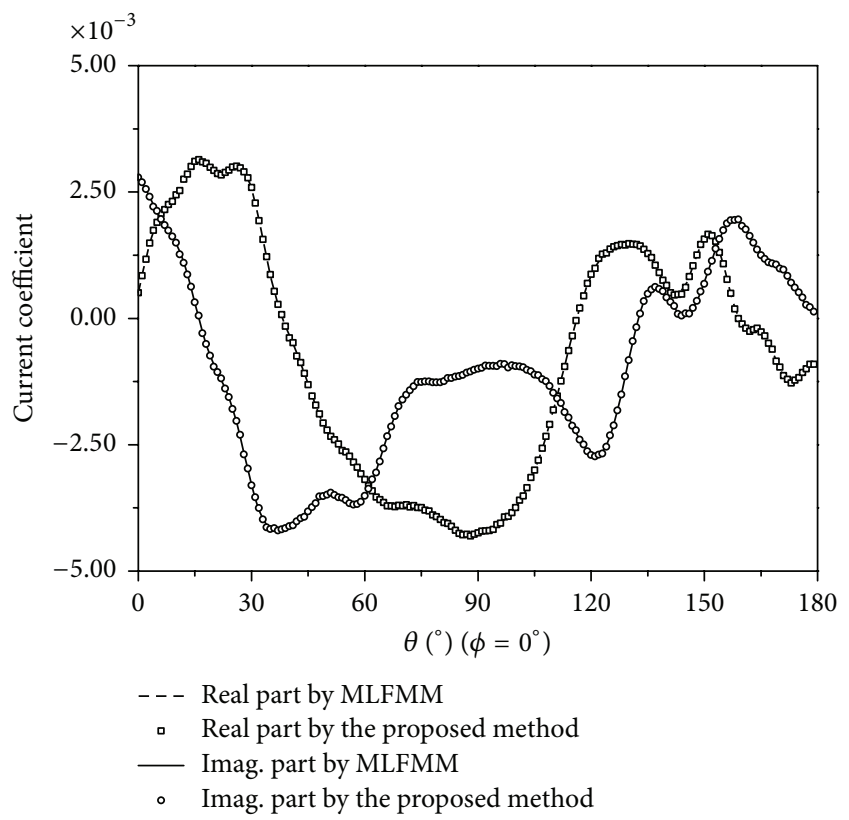

FIGURE 6: True and recovery current coefficients for missile model.

MoM technique has been presented. A hybrid compressive method using the CS technique and the ACA is introduced to compress the excitation matrix. The numerical results demonstrate that the adopted approach leads to very accurate

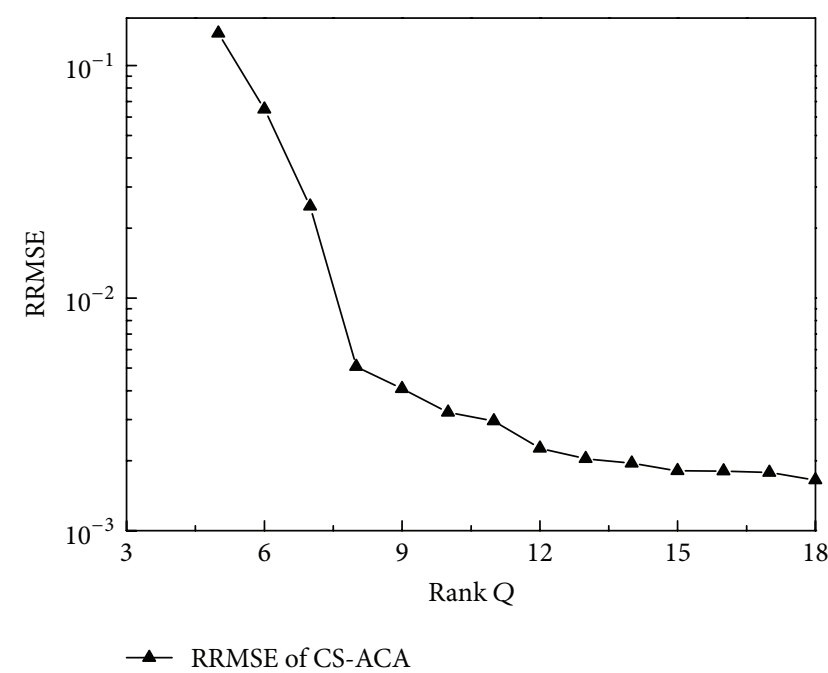

FIgURE 7: RRMSE for missile model.

RCS results. Furthermore, the ratio of the number of RHS between the compressed excitation matrix and the original excitation matrix in the conventional MoM is close to one to fifteen.

\section{Competing Interests}

The authors declare that they have no competing interests.

\section{Acknowledgments}

This work was supported by the National Natural Science Foundation of China (Grant no. 61172020), the Specialized Research Fund for the Doctoral Program of Higher Education of China (Grant no. 20123401110006), and the Fund for 211 Project of Anhui University, China (Grant no. ZYGG201202).

\section{References}

[1] R. F. Harrington, Field Computation by Method of Moments, Macmillan, New York, NY, USA, 1968.

[2] W. C. Gibson, The Method of Moments in Electromagnetics, CRC Press, Boca Raton, Fla, USA, 2007.

[3] J. Song, C. C. Lu, and W. C. Chew, "Multilevel fast multipole algorithm for electromagnetic scattering by large complex objects," IEEE Transactions on Antennas and Propagation, vol. 45, no. 10, pp. 1488-1493, 1997.

[4] E. Bleszynski, M. Bleszynski, and T. Jaroszewicz, "AIM: adaptive integral method for solving large-scale electromagnetic scattering and radiation problems," Radio Science, vol. 31, no. 5, pp. 1225-1251, 1996.

[5] M. Bebendorf, "Approximation of boundary element matrices," Numerische Mathematik, vol. 86, no. 4, pp. 565-589, 2000.

[6] K. Zhao, M. N. Vouvakis, and J.-F. Lee, "The adaptive cross approximation algorithm for accelerated method of moments computations of EMC problems," IEEE Transactions on Electromagnetic Compatibility, vol. 47, no. 4, pp. 763-773, 2005. 
[7] M. S. Chen, F. L. Liu, H. M. Du, and X. L. Wu, "Compressive sensing for fast analysis of wide-angle monostatic scattering problems," IEEE Antennas and Wireless Propagation Letters, vol. 10, no. 3, pp. 1243-1246, 2014.

[8] X.-Y. Cao, M.-S. Chen, and X.-L. Wu, "Application of compressed sensing to solving $3 \mathrm{D}$ electromagnetic scattering problems over a wide angle," in Proceedings of the International Conference on Microwave and Millimeter Wave Technology (ICMMT '12), pp. 720-723, Shenzhen, China, May 2012.

[9] R. G. Baraniuk, “Compressive sensing," IEEE Signal Processing Magazine, vol. 24, no. 4, pp. 118-121, 2007.

[10] R. G. Baraniuk, V. Cevher, M. F. Duarte, and C. Hegde, "Modelbased compressive sensing," Institute of Electrical and Electronics Engineers. Transactions on Information Theory, vol. 56, no. 4, pp. 1982-2001, 2010.

[11] L. Carin, D. Liu, W. Lin, and B. Guo, "Compressive sensing for multi-static scattering analysis," Journal of Computational Physics, vol. 228, no. 9, pp. 3464-3477, 2009.

[12] T. T. Cai and L. Wang, "Orthogonal matching pursuit for sparse signal recovery with noise," IEEE Transactions on Information Theory, vol. 57, no. 7, pp. 4680-4688, 2011.

[13] E. J. Candés, "Compressive sampling," in Proceedings of the International Congress of Mathematicians, pp. 1433-1452, European Mathematical Society, Madrid, Spain, 2006.

[14] D. L. Donoho, "Compressed sensing," IEEE Transactions on Information Theory, vol. 52, no. 4, pp. 1289-1306, 2006.

[15] E. J. Candés, J. Romberg, and T. Tao, "Robust uncertainty principles: exact signal reconstruction from highly incomplete frequency information," IEEE Transactions on Information Theory, vol. 52, no. 2, pp. 489-509, 2006.

[16] S. M. Rao, D. R. Wilton, and A. W. Glisson, "Electromagnetic scattering by surfaces of arbitrary shape," IEEE Transactions on Antennas and Propagation, vol. 30, no. 3, pp. 409-418, 1982.

[17] J. Shaeffer, "Direct solve of electrically large integral equations for problem sizes to $1 \mathrm{M}$ unknowns," IEEE Transactions on Antennas and Propagation, vol. 56, no. 8, pp. 2306-2313, 2008.

[18] A. C. Woo, H. T. G. Wang, M. J. Schuh, and M. L. Sanders, "Benchmark radar targets for the validation of computational electromagnetics programs," IEEE Transactions on Antennas and Propagation, vol. 35, no. 1, pp. 84-89, 1993.

[19] R. Cicchetti, D. Caratelli, and A. Faraone, "Exact closed-form expression of the electromagnetic field excited by a uniform current distribution lying on a Cartesian quadrant," IEEE Transactions on Antennas and Propagation, vol. 61, no. 4, pp. 21422155, 2013. 


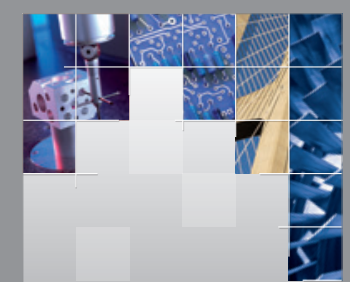

\section{Enfincering}
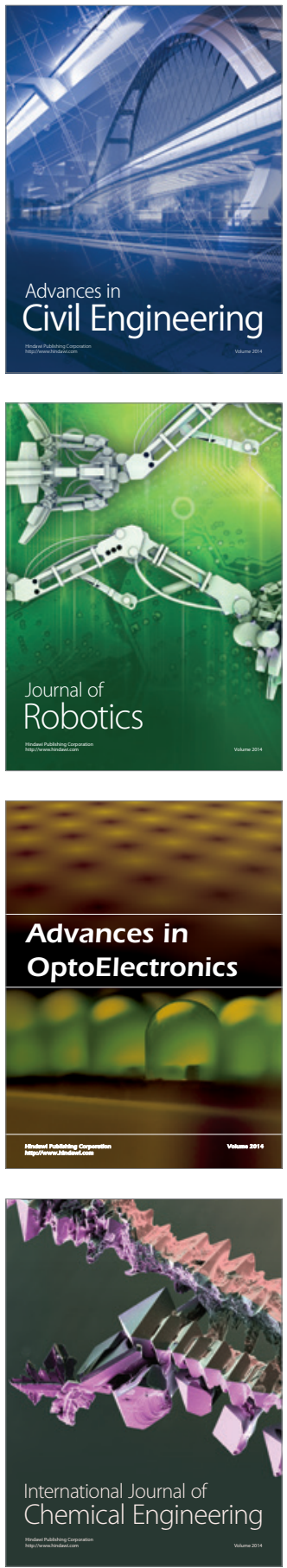

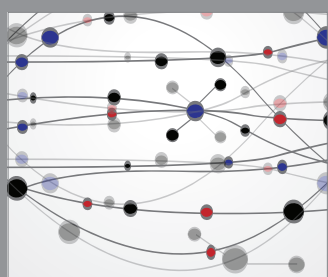

The Scientific World Journal

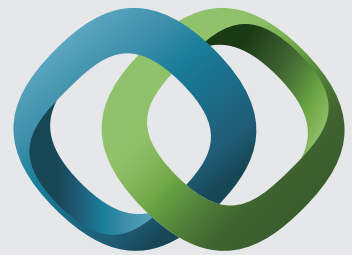

\section{Hindawi}

Submit your manuscripts at

http://www.hindawi.com
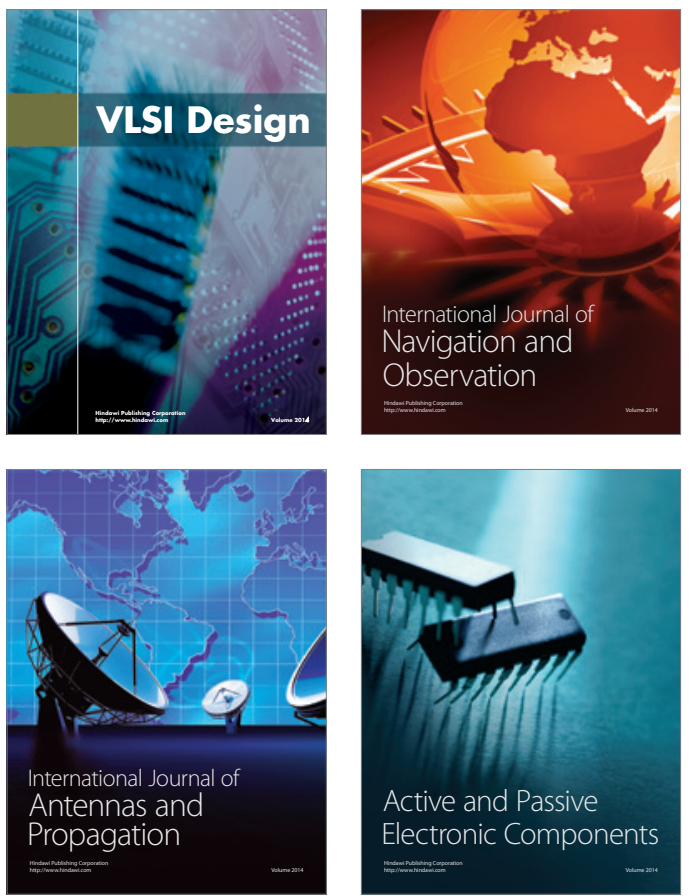
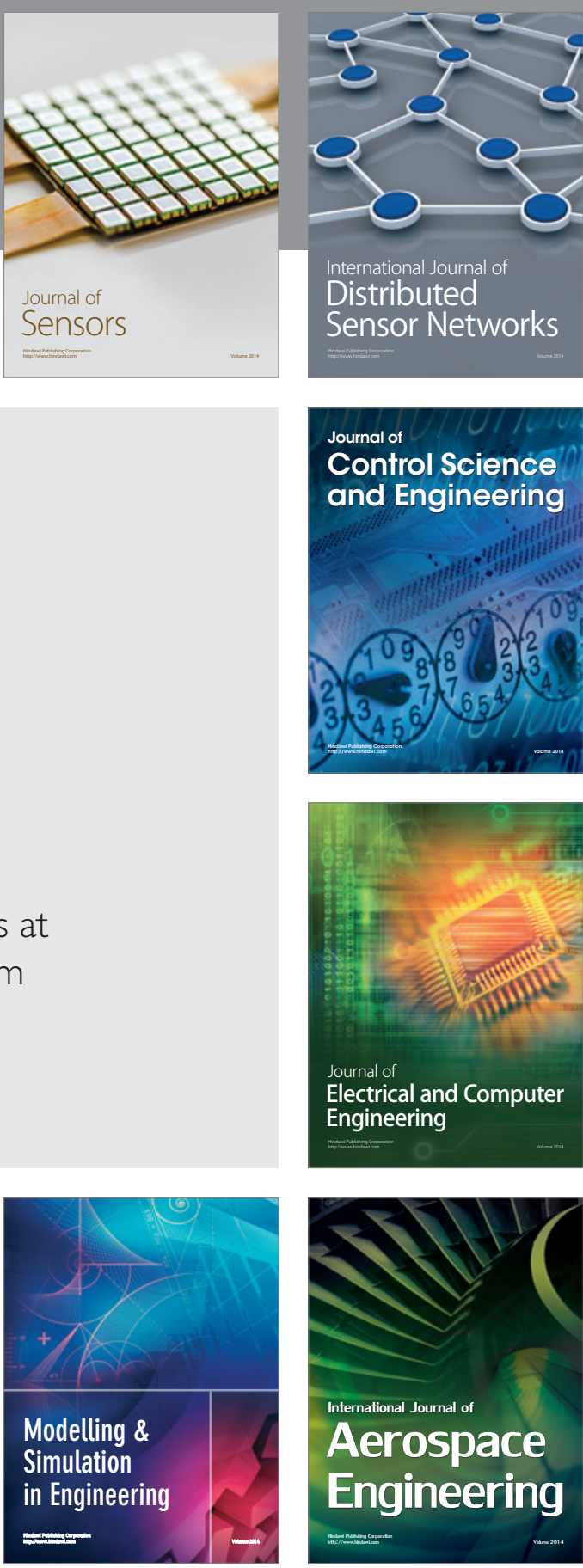

International Journal of

Distributed

Sensor Networks

Journal of

Control Science

and Engineering
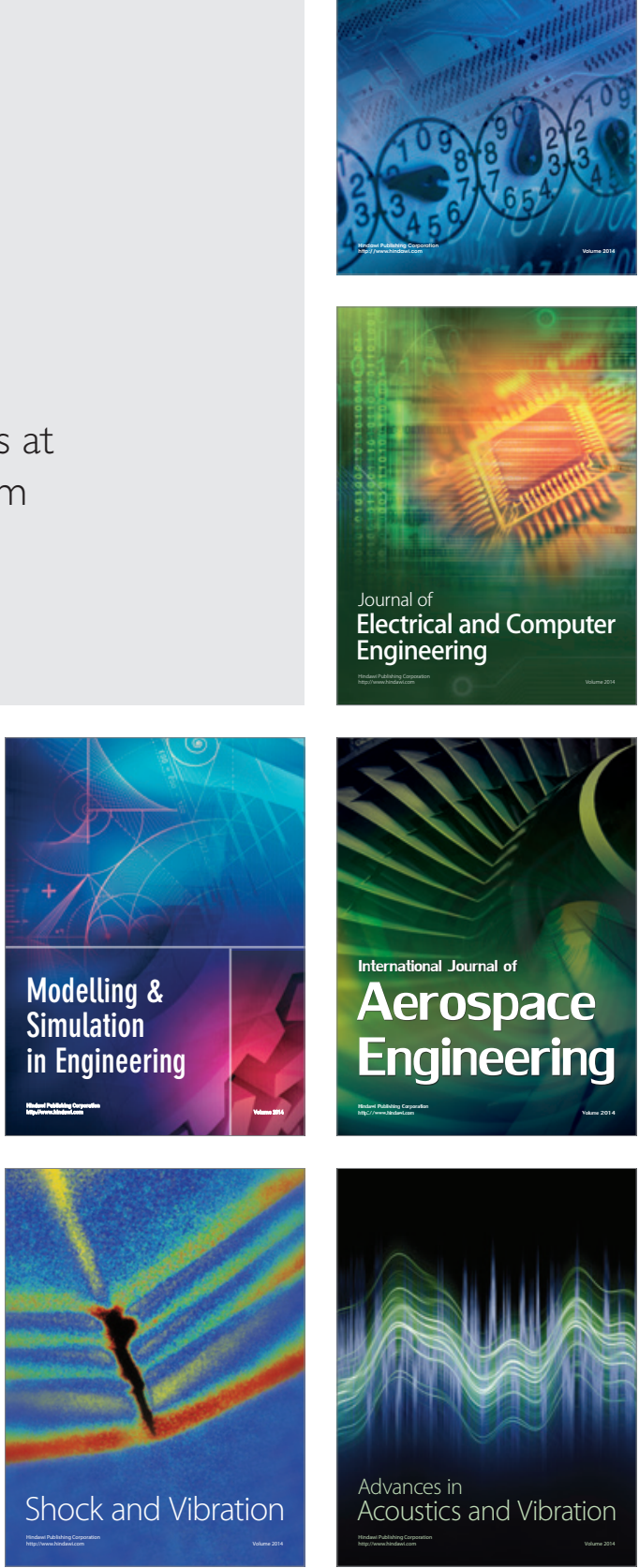